\title{
First-principles effective-mass Hamiltonian for semiconductor nanostructures in a magnetic field
}

\author{
Bradley A Foreman \\ Department of Physics, Hong Kong University of Science and Technology, Clear Water Bay, \\ Kowloon, Hong Kong, People's Republic of China \\ E-mail: phbaf@ust.hk
}

Received 30 June 2005, in final form 19 September 2005

Published 11 January 2006

Online at stacks.iop.org/JPhysCM/18/1335

\begin{abstract}
A multi-band effective-mass Hamiltonian is derived for lattice-matched semiconductor nanostructures in a slowly varying external magnetic field. The theory is derived from the first-principles magnetic-field coupling Hamiltonian of Pickard and Mauri, which is applicable to nonlocal norm-conserving pseudopotentials in the local density approximation to density functional theory. The pseudopotential of the nanostructure is treated as a perturbation of a bulk reference crystal, with linear and quadratic response terms included in $\boldsymbol{k} \cdot \boldsymbol{p}$ perturbation theory. The resulting Hamiltonian contains several interface terms that have not been included in previous envelope-function theories for nanostructures in a magnetic field. The derivation provides the first direct analytical expressions showing how the coupling of the nonlocal potential to the magnetic field influences the effective magnetic dipole moment of the electron.
\end{abstract}

\section{Introduction}

Recent experiments on the spin Hall effect [1,2] and the spin galvanic and circular photogalvanic effects $[3,4]$ have stimulated renewed interest in the behaviour of electrons and holes in semiconductor nanostructures in an external magnetic field. Such experiments are often modelled using an effective-mass or envelope-function Hamiltonian [5-7], which may include a generalized Rashba coupling [8] to account for spin-splitting effects. The magnetic field is included via the minimal coupling substitution $k \rightarrow k+A / c$, which is well justified for bulk semiconductors [9-11].

However, Mlinar et al [12] have recently demonstrated that the results of such calculations can depend strongly on the particular choice of envelope-function Hamiltonian. The standard 'symmetrized' Hamiltonian [13] applied to GaAs/AlAs and InAs/GaAs quantum dots in a strong magnetic field yielded energy eigenstates with arguably unphysical properties [12] in contrast with the physically reasonable predictions [12] obtained from an asymmetric 
Hamiltonian $[14,15]$. This may be viewed as a modern confirmation of Luttinger's original work [10] demonstrating the importance of including antisymmetric terms in the Hamiltonian.

Nevertheless, it is not clear that the model used by Mlinar et al [12] provides a correct description of the heterojunction regions. The antisymmetric part of the valence-band Hamiltonian derived in reference [14] is in fact just the valence-band Rashba coupling [8] that is of interest for spintronics applications [16]. However, a recent first-principles envelopefunction theory for lattice-matched heterostructures [17] has established the existence of several other interface terms of the same order of magnitude as the Rashba term. These terms are not included in standard effective-mass models for nanostructures, although some authors [18] have included phenomenological surface terms in their study of the magnetic field problem.

The purpose of this paper is to establish a rigorous theoretical foundation for the effectivemass theory of semiconductor nanostructures in a magnetic field. This is done by extending the first-principles multi-band effective-mass Hamiltonian of [17] to include the effects of a slowly varying static external magnetic field. In this theory [17], the nanostructure pseudopotential is treated as a perturbation of a bulk reference crystal (e.g., $\mathrm{Al}_{0.5} \mathrm{Ga}_{0.5} \mathrm{As}$ for a $\mathrm{GaAs} / \mathrm{AlAs}$ quantum dot), with the self-consistent linear and quadratic response terms included in the framework of the $\boldsymbol{k} \cdot \boldsymbol{p}$ perturbation theory of Leibler [19].

Until recently, such a first-principles analysis would not have been possible, due to the lack of a generally accepted Hamiltonian to describe the coupling between the magnetic field and the nonlocal part of the potential energy. Such nonlocal terms arise for two reasons. At the fundamental level, the self-energy operator (which accounts for electron-electron Coulomb interactions) in Dyson's equation is nonlocal. At the practical level, the pseudopotentials used in most first-principles calculations (which account for the influence of core electrons) are also nonlocal.

Pickard and Mauri [20] have solved the second problem by deriving a coupling for normconserving pseudopotentials in the local density approximation (LDA) [21] that reproduces the results of all-electron LDA calculations. Ismail-Beigi et al [22] have proposed a more general coupling for arbitrary nonlocal potentials, but since this coupling, in the special case of normconserving pseudopotentials, does not agree with all-electron calculations [20], its validity in the general case is questionable.

The present work is therefore limited to the case of norm-conserving pseudopotentials within the LDA. Although LDA calculations do not accurately predict energy gaps in semiconductors, this at least provides a working model that is self-consistent and accounts for the true atomic structure of a heterointerface. The results obtained here should be representative of the general features obtained in a more complete theory that includes the nonlocal quasiparticle self-energy.

This paper, like the previous work [17] upon which it is based, is applicable to latticematched semiconductor nanostructures of arbitrary shape (e.g., quantum wells, wires, or dots), but with dimensions such that the slowly varying envelope approximation is valid (see section 3 below). For simplicity, it is assumed that the atoms occupying equivalent lattice sites of the reference crystal have the same chemical valence (i.e., the nanostructure is generated by isovalent substitutions), and that the symmetry of the reference crystal does not support a spontaneous polarization (thus, wurtzite materials are not included). These assumptions are not fundamental limitations of the theory [23]; they are merely used to simplify the form of the resulting Hamiltonian. Examples of the material systems that could be described by this theory include the technologically important zinc-blende materials GaAs/AlAs, InAs/GaSb, and $\mathrm{In}_{0.53} \mathrm{Ga}_{0.47} \mathrm{As} / \mathrm{InP}$ (the alloying in the latter being treated in the virtual crystal approximation). Although lattice mismatch is not included in this theory, a first-principles linear-response treatment of strain has been shown to yield good results for band offsets in strained $\mathrm{Si} / \mathrm{Ge}$ 
heterostructures $[24,25]$, so it should be possible to extend the present perturbative analysis to the case of lattice-mismatched systems in future work.

The results derived in this paper confirm the existence of interface terms in the effectivemass Hamiltonian that were not included in the calculations of Mlinar et al [12]. An explicit numerical calculation of these terms is in development and will be reported elsewhere. However, the present analytical results show that these terms are of the same order of magnitude as the valence-band Rashba terms studied in [12], which suggests that they may have a comparable influence on the energy eigenvalues and eigenstates in an external magnetic field.

In addition, an analytical demonstration of the difference between the coupling Hamiltonians of Pickard and Mauri [20] and Ismail-Beigi et al [22] is established for the first time. In particular, the nonlocal pseudopotential contributes to the effective magnetic dipole moment of the electron in the first case, while in the second case it does not.

The paper begins in section 2 with the derivation of exact expressions for the magnetic-field coupling Hamiltonian in coordinate and momentum space. Approximations suitable for slowly varying envelope functions are developed in section 3 , based on a power series expansion of the nonlocal pseudopotential in momentum space. These results are used to construct an envelopefunction Hamiltonian in section 4 . The contributions derived from $\boldsymbol{k} \cdot \boldsymbol{p}$ perturbation theory are added in section 5, where the complete expression for the effective-mass Hamiltonian is given. The results are discussed in the concluding section 6 . Throughout the paper, Hartree atomic units $(\hbar=m=e=1)$ are used.

\section{General form of the coupling Hamiltonian}

The magnetic coupling Hamiltonian derived by Pickard and Mauri [20] is applicable to nonlocal potentials formed by a superposition of localized nonoverlapping norm-conserving pseudopotentials; i.e.,

$$
V^{\mathrm{nl}}\left(\boldsymbol{x}, \boldsymbol{x}^{\prime}\right)=\sum_{R} V_{R}^{\mathrm{nl}}\left(\boldsymbol{x}, \boldsymbol{x}^{\prime}\right)
$$

where $V_{\boldsymbol{R}}^{\mathrm{nl}}\left(\boldsymbol{x}, \boldsymbol{x}^{\prime}\right)$ is the nonlocal part of the pseudopotential for an ion at position $\boldsymbol{R}$. In this case, the potential in the presence of a slowly varying magnetic field $\boldsymbol{B}(\boldsymbol{x})=\nabla \times \boldsymbol{A}(\boldsymbol{x})$ is given by [20]

$$
\tilde{V}^{\mathrm{nl}}\left(\boldsymbol{x}, \boldsymbol{x}^{\prime}\right)=\sum_{\boldsymbol{R}} V_{\boldsymbol{R}}^{\mathrm{nl}}\left(\boldsymbol{x}, \boldsymbol{x}^{\prime}\right) \exp \left(\frac{\mathrm{i}}{c} \int_{\boldsymbol{x} \rightarrow \boldsymbol{R} \rightarrow \boldsymbol{x}^{\prime}} \boldsymbol{A}\left(\boldsymbol{x}^{\prime \prime}\right) \cdot \mathrm{d} \boldsymbol{x}^{\prime \prime}\right),
$$

where $\boldsymbol{x} \rightarrow \boldsymbol{R}$ denotes an integral over the straight-line path from $\boldsymbol{x}$ to $\boldsymbol{R}$. If $\boldsymbol{A}(\boldsymbol{x})$ is chosen to be periodic $[11,20,26]$, it can be expanded in a Fourier series, which permits the line integral to be evaluated as

$$
\int_{\boldsymbol{R} \rightarrow \boldsymbol{x}^{\prime}} \boldsymbol{A} \cdot \mathrm{d} \boldsymbol{x}^{\prime \prime}=\left(\boldsymbol{x}^{\prime}-\boldsymbol{R}\right) \cdot \sum_{\boldsymbol{k}} \boldsymbol{A}(\boldsymbol{k}) \mathrm{e}^{\mathrm{i} k \cdot \boldsymbol{R}} f\left[\mathrm{i} \boldsymbol{k} \cdot\left(\boldsymbol{x}^{\prime}-\boldsymbol{R}\right)\right],
$$

in which

$$
f(x)=\frac{\mathrm{e}^{x}-1}{x}=1+\frac{x}{2 !}+\frac{x^{2}}{3 !}+\cdots .
$$

Since the coupling (2) is valid only for slowly varying $\boldsymbol{B}(\boldsymbol{x})$ [20], it is assumed that $\boldsymbol{A}(\boldsymbol{k})$ is significant only for small $k$ (i.e., $k a \ll 1$, where $a$ is the lattice constant). The power series in (4) therefore converges rapidly, because the range of the nonlocal potential (i.e., the core 
radius) is less than $a$. From this power series one obtains

$$
\begin{aligned}
\int_{\boldsymbol{R} \rightarrow \boldsymbol{x}^{\prime}} \boldsymbol{A} \cdot \mathrm{d} \boldsymbol{x}^{\prime \prime} & =\left(\boldsymbol{x}^{\prime}-\boldsymbol{R}\right) \cdot \boldsymbol{A}(\boldsymbol{R})+\frac{1}{2 !}\left(x_{\lambda}^{\prime}-R_{\lambda}\right)\left(x_{\mu}^{\prime}-R_{\mu}\right) \frac{\partial A_{\lambda}}{\partial R_{\mu}} \\
& +\frac{1}{3 !}\left(x_{\lambda}^{\prime}-R_{\lambda}\right)\left(x_{\mu}^{\prime}-R_{\mu}\right)\left(x_{\nu}^{\prime}-R_{\nu}\right) \frac{\partial^{2} A_{\lambda}}{\partial R_{\mu} \partial R_{\nu}}+\cdots,
\end{aligned}
$$

in which a sum over repeated Cartesian coordinate indices $\lambda, \mu, v$ is assumed, and $\partial A_{\lambda} / \partial R_{\mu} \equiv$ $\left.\left(\partial A_{\lambda} / \partial x_{\mu}\right)\right|_{x=R}$. Therefore

$$
\begin{aligned}
\int_{x \rightarrow \boldsymbol{R} \rightarrow x^{\prime}} \boldsymbol{A} \cdot \mathrm{d} \boldsymbol{x}^{\prime \prime}=\left(\boldsymbol{x}^{\prime}-\boldsymbol{x}\right) \cdot \boldsymbol{A}(\boldsymbol{R}) \\
+\frac{1}{2 !}\left[\left(x_{\lambda}^{\prime}-R_{\lambda}\right)\left(x_{\mu}^{\prime}-R_{\mu}\right)-\left(x_{\lambda}-R_{\lambda}\right)\left(x_{\mu}-R_{\mu}\right)\right] \frac{\partial A_{\lambda}}{\partial R_{\mu}}+\cdots
\end{aligned}
$$

Note that equations (5) and (6) depend only on the symmetric part of the matrix $\partial A_{\lambda} / \partial R_{\mu}$; i.e., they contain no terms proportional to $\boldsymbol{B}$ or its derivatives. As shown in the appendix, this is generally not true for other coupling Hamiltonians.

For the potential energy of a lattice-matched heterostructure, it is convenient to use a more explicit notation:

$$
V\left(\boldsymbol{x}, \boldsymbol{x}^{\prime}\right)=\sum_{\alpha, \boldsymbol{R}} f_{\boldsymbol{R}}^{\alpha} v^{\alpha}\left(\boldsymbol{x}-\boldsymbol{R}^{\alpha}, \boldsymbol{x}^{\prime}-\boldsymbol{R}^{\alpha}\right)
$$

in which $\boldsymbol{R}$ is now a Bravais lattice vector (for the periodic bulk reference crystal), $\alpha$ labels an atom at position $\boldsymbol{\tau}^{\alpha}$ in the unit cell, and $\boldsymbol{R}^{\alpha}=\boldsymbol{R}+\boldsymbol{\tau}^{\alpha}$. The function $f_{\boldsymbol{R}}^{\alpha}$ is the atomic mole fraction of atom $\alpha$ at position $\boldsymbol{R}^{\alpha}$, while $v^{\alpha}\left(\boldsymbol{x}, \boldsymbol{x}^{\prime}\right)$ is the nonlocal ionic pseudopotential for atom $\alpha$.

With the coupling to the magnetic field given by equations (2) and (6), the Fourier transform of the field-dependent potential can be written as

$$
\tilde{V}\left(\boldsymbol{k}, \boldsymbol{k}^{\prime}\right)=\sum_{\alpha, \boldsymbol{R}} f_{\boldsymbol{R}}^{\alpha} \mathrm{e}^{-\mathrm{i}\left(\boldsymbol{k}-\boldsymbol{k}^{\prime}\right) \cdot \boldsymbol{R}^{\alpha}} \exp \left[\mathrm{i} \xi\left(\hat{\boldsymbol{x}}, \hat{\boldsymbol{x}}^{\prime}\right) / c\right] v^{\alpha}\left(\boldsymbol{K}, \boldsymbol{K}^{\prime}\right),
$$

in which $\boldsymbol{K}$ is the kinetic momentum

$$
\boldsymbol{K}=\boldsymbol{k}+\frac{1}{c} \boldsymbol{A}\left(\boldsymbol{R}^{\alpha}\right), \quad \boldsymbol{K}^{\prime}=\boldsymbol{k}^{\prime}+\frac{1}{c} \boldsymbol{A}\left(\boldsymbol{R}^{\alpha}\right) .
$$

The operator function $\xi$ is defined by

$\xi\left(\hat{\boldsymbol{x}}, \hat{\boldsymbol{x}}^{\prime}\right)=\frac{1}{2 !}\left(\hat{x}_{\lambda}^{\prime} \hat{x}_{\mu}^{\prime}-\hat{x}_{\lambda} \hat{x}_{\mu}\right) \frac{\partial A_{\lambda}}{\partial R_{\mu}^{\alpha}}+\frac{1}{3 !}\left(\hat{x}_{\lambda}^{\prime} \hat{x}_{\mu}^{\prime} \hat{x}_{v}^{\prime}-\hat{x}_{\lambda} \hat{x}_{\mu} \hat{x}_{\nu}\right) \frac{\partial^{2} A_{\lambda}}{\partial R_{\mu}^{\alpha} \partial R_{v}^{\alpha}}+\cdots$,

in which $\hat{x}_{\lambda}=\mathrm{i} \partial / \partial k_{\lambda}$ and $\hat{x}_{\mu}^{\prime}=-\mathrm{i} \partial / \partial k_{\mu}^{\prime}$. These expressions are used to develop an effectivemass Hamiltonian in what follows.

\section{Approximations to the Hamiltonian}

Effective-mass theory is based on approximations valid for $k_{\text {env }} a \ll 1$, where $k_{\text {env }}$ is a typical wavenumber for a slowly varying envelope function. This condition will hold, for example, in a wide quantum well of width $L \gg a$, for which $k_{\mathrm{env}} \sim 2 \pi / L$. The external magnetic field $B$ must also be weak enough for the magnetic length $L_{B}=\sqrt{c / B}$ to satisfy $L_{B} \gg a$ [11].

In addition to these standard conditions, if $\boldsymbol{K}$ is to be treated as a small quantity of the same order as $k$, the vector potential must be small enough to satisfy $a A / c \ll 1$. For a sinusoidal field with $B \sim k_{B} A$ [26], where $k_{B}$ is the wavenumber of the external field, this condition 
is equivalent to $k_{B} L_{B}^{2} \gg a$. Combining this with the previous condition $L_{B} \gg a$, the new condition will be satisfied if $k_{B} L_{B} \gtrsim 1$. Thus, very small values of $k_{B}$ place an additional constraint on $L_{B}$. This constraint is not usually mentioned [11], but it is implicit in any effective-mass treatment where $\boldsymbol{K}$ is assumed to be of the same order as $\boldsymbol{k}$. Therefore, effectivemass calculations for truly constant $\boldsymbol{B}$ fields (which are obtained in the limit $k_{B} \rightarrow 0$ [26]) can only yield results that are valid asymptotically in the limit $B \rightarrow 0$. The asymptotic nature of effective-mass theory for constant $\boldsymbol{B}$ was suggested by Kohn [27], and stated more explicitly by Foldy and Wouthuysen [28] in connection with the nonrelativistic approximation to the Dirac equation.

In the Luttinger-Kohn representation $|n \boldsymbol{k}\rangle$ [9], the wavefunction $\langle n \boldsymbol{k} \mid \psi\rangle$ is just a unitary transformation of the momentum-space wavefunction $\langle\boldsymbol{k}+\boldsymbol{G} \mid \psi\rangle$ [29]:

$$
\langle n k \mid \psi\rangle=\sum_{G} U_{n G}^{\dagger}\langle\boldsymbol{k}+\boldsymbol{G} \mid \psi\rangle .
$$

Here $G$ is a reciprocal lattice vector of the reference crystal, and the spinor $U_{n} G$ is a Fourier series coefficient for the $n$th zone-centre Bloch function of the reference crystal. For slowly varying envelope functions $F_{n}(\boldsymbol{x})$, the Fourier transform $F_{n}(\boldsymbol{k}) \equiv\langle n \boldsymbol{k} \mid \psi\rangle$ (and hence $\langle\boldsymbol{k}+\boldsymbol{G} \mid \psi\rangle$ ) is significant only for small $k$. (A review of the numerical evidence supporting the use of the slowly varying envelope approximation in abrupt heterostructures is given in [17].) Thus, slowly varying envelope functions probe the nonlocal pseudopotential only within a small neighbourhood of the reciprocal lattice vectors $G$. Within this neighbourhood, the ionic pseudopotential can be approximated by the leading terms in its Taylor series expansion [17]:

$$
\begin{aligned}
v^{\alpha}\left(\boldsymbol{k}+\boldsymbol{G}, \boldsymbol{k}^{\prime}\right. & \left.+\boldsymbol{G}^{\prime}\right)=v_{G G^{\prime}}^{(\cdot \mid \cdot) \alpha}+k_{\lambda} v_{G G^{\prime}}^{(\lambda \mid \cdot) \alpha}+k_{\lambda}^{\prime} v_{G G^{\prime}}^{(\cdot \mid \lambda) \alpha}+k_{\lambda} k_{\mu} v_{G G^{\prime}}^{(\lambda \mu \mid \cdot) \alpha}+k_{\lambda}^{\prime} k_{\mu}^{\prime} v_{G G^{\prime}}^{(\cdot \mid \lambda \mu) \alpha} \\
& +k_{\lambda} k_{\mu}^{\prime} v_{G G^{\prime}}^{(\lambda \mid \mu) \alpha}+\cdots,
\end{aligned}
$$

where $v_{G G^{\prime}}^{(\lambda \mid \mu) \alpha}$ is an expansion coefficient. This expansion is valid because $v^{\alpha}\left(\boldsymbol{x}, \boldsymbol{x}^{\prime}\right)$ is localized, hence $v^{\alpha}\left(\boldsymbol{k}, \boldsymbol{k}^{\prime}\right)$ is analytic. For simplicity, terms beyond the second order are not written out explicitly here; however, the present effective-mass theory [17] retains terms of order $k^{4}$ in the Hamiltonian of the reference crystal (see equation (17) below).

By definition, the expansion coefficients are symmetric with respect to arbitrary permutations of the indices on one side of the vertical line-for example, $v_{G G^{\prime}}^{(\lambda \mu \mid \cdot) \alpha}=v_{G G^{\prime}}^{(\mu \lambda \mid \cdot)}$. However, $v_{G G^{\prime}}^{(\lambda \mid \mu) \alpha} \neq v_{G G^{\prime}}^{(\mu \mid \lambda) \alpha}$. For indices on opposite sides of the vertical line, the only symmetries are those obtained from hermiticity and time reversal, such as $v_{G G^{\prime}}^{(\lambda \mid \mu) \alpha}=\left(v_{G^{\prime} G}^{(\mu \mid \lambda) \alpha}\right)^{\dagger}$ and $v_{G G^{\prime}}^{(\cdot \mid \lambda) \alpha}=\left(v_{G^{\prime} G}^{(\lambda \mid \cdot) \alpha}\right)^{\dagger}$.

With the above restrictions on the magnetic field, $\boldsymbol{K}$ is a small quantity of the same order as $\boldsymbol{k}$, and the potential (8) can be expanded in a Taylor series about the reciprocal lattice vectors $G$. This yields

$$
\begin{aligned}
\tilde{V}\left(\boldsymbol{k}+\boldsymbol{G}, \boldsymbol{k}^{\prime}+\right. & \left.\boldsymbol{G}^{\prime}\right)=\sum_{\alpha, \boldsymbol{R}} f_{\boldsymbol{R}^{\alpha}}^{\alpha} \mathrm{e}^{-\mathrm{i}\left(\boldsymbol{k}-\boldsymbol{k}^{\prime}\right) \cdot \boldsymbol{R}^{\alpha}} \mathrm{e}^{-\mathrm{i}\left(\boldsymbol{G}-\boldsymbol{G}^{\prime}\right) \cdot \tau^{\alpha}}\left[v_{G G^{\prime}}^{(\cdot \cdot) \alpha}+K_{\lambda} v_{G G^{\prime}}^{(\lambda \mid \cdot) \alpha}+K_{\lambda}^{\prime} v_{G G^{\prime}}^{(\cdot \mid \lambda) \alpha}\right. \\
& +\left(K_{\lambda} K_{\mu}+\frac{\mathrm{i}}{c} \frac{\partial A_{\lambda}}{\partial R_{\mu}^{\alpha}}\right) v_{G G^{\prime}}^{(\lambda \mu \mid \cdot) \alpha}+\left(K_{\lambda}^{\prime} K_{\mu}^{\prime}-\frac{\mathrm{i}}{c} \frac{\partial A_{\lambda}}{\partial R_{\mu}^{\alpha}}\right) v_{G G^{\prime}}^{(\cdot \mid \lambda \mu) \alpha} \\
& \left.+K_{\lambda} K_{\mu}^{\prime} v_{G G^{\prime}}^{(\lambda \mid \mu) \alpha}+\cdots\right],
\end{aligned}
$$

where the expansion coefficients are the same as in (12), and the terms involving $\partial A_{\lambda} / \partial R_{\mu}^{\alpha}$ are the leading contributions from (10). 


\section{Envelope-function Hamiltonian}

These results are now used to construct an envelope-function Hamiltonian valid for slowly varying envelopes. In the Luttinger-Kohn representation, the matrix elements of $\tilde{V}$ are given by the unitary transformation

$$
\tilde{V}_{n n^{\prime}}\left(\boldsymbol{k}, \boldsymbol{k}^{\prime}\right)=\sum_{\boldsymbol{G}, \boldsymbol{G}^{\prime}} U_{n \boldsymbol{G}}^{\dagger} \tilde{V}\left(\boldsymbol{k}+\boldsymbol{G}, \boldsymbol{k}^{\prime}+\boldsymbol{G}^{\prime}\right) U_{n^{\prime} \boldsymbol{G}^{\prime}} .
$$

When the expansion (13) is inserted into equation (14), one obtains transformed expansion coefficients such as

$$
V_{n n^{\prime}}^{(\lambda \mu \mid \cdot) \alpha}=N \sum_{G, G^{\prime}} U_{n G}^{\dagger} v_{G G^{\prime}}^{(\lambda \mu \mid \cdot) \alpha} U_{n^{\prime} G^{\prime}} \mathrm{e}^{-\mathrm{i}\left(G-G^{\prime}\right) \cdot \tau^{\alpha}}
$$

in which $N$ is the number of unit cells in the crystal. It is also convenient to introduce coordinate-space matrix elements of the form

$$
V_{n n^{\prime}}^{(\lambda \mu \mid \cdot)}(\boldsymbol{x})=\sum_{\alpha} V_{n n^{\prime}}^{(\lambda \mu \mid \cdot) \alpha} f^{\alpha}(\boldsymbol{x})
$$

in which $f^{\alpha}(x)$ is a macroscopic average (see [17]) of the discrete atomic distribution function $f_{\boldsymbol{R}}^{\alpha}$. Note that $V_{n n^{\prime}}^{(\lambda \mu \mid \cdot)}(\boldsymbol{x})=V_{n n^{\prime}}^{(\mu \lambda \mid \cdot)}(\boldsymbol{x})=\left[V_{n^{\prime} n}^{(\cdot \mid \lambda \mu)}(\boldsymbol{x})\right]^{*}$ and $V_{n n^{\prime}}^{(\lambda \mid \mu)}(\boldsymbol{x})=\left[V_{n^{\prime} n}^{(\mu \mid \lambda)}(\boldsymbol{x})\right]^{*}$.

With these notational conventions, the Fourier transform of the matrix element (14) yields the following contribution from the nonlocal pseudopotential to the envelope-function Hamiltonian:

$$
\begin{aligned}
\tilde{V}_{n n^{\prime}}(\boldsymbol{x}, \boldsymbol{p})= & V_{n n^{\prime}}^{(\cdot \cdot)}(\boldsymbol{x})+P_{\lambda} V_{n n^{\prime}}^{(\lambda \mid \cdot)}(\boldsymbol{x})+V_{n n^{\prime}}^{(\cdot \mid \lambda)}(\boldsymbol{x}) P_{\lambda}+P_{\lambda} P_{\mu} V_{n n^{\prime}}^{(\lambda \mu \mid \cdot)}(\boldsymbol{x})+V_{n n^{\prime}}^{(\cdot \mid \lambda \mu)}(\boldsymbol{x}) P_{\lambda} P_{\mu} \\
& +P_{\lambda} V_{n n^{\prime}}^{(\lambda \mid \mu)}(\boldsymbol{x}) P_{\mu}+V_{n n^{\prime}}^{\lambda \mu \nu} P_{\lambda} P_{\mu} P_{\nu}+V_{n n^{\prime}}^{\lambda \mu \kappa v} P_{\lambda} P_{\mu} P_{\kappa} P_{\nu},
\end{aligned}
$$

in which the canonical and kinetic momentum operators are $\boldsymbol{p}=-\mathrm{i} \nabla$ and $\boldsymbol{P}=\boldsymbol{p}+\boldsymbol{A}(\boldsymbol{x}) / c$. The derivation of this result makes use of the usual approximations [11] for slowly varying potentials $\boldsymbol{A}(\boldsymbol{x})$ and envelope functions $F_{n}(\boldsymbol{x})$. In other words, it was assumed that since only small values of $k$ are of interest, the error involved in replacing the Fourier transform of the discrete product $f_{\boldsymbol{R}}^{\alpha} \boldsymbol{A}\left(\boldsymbol{R}_{\alpha}\right)$ with the Fourier transform of the continuous product $f^{\alpha}(\boldsymbol{x}) \boldsymbol{A}(\boldsymbol{x})$ is negligible, and the upper limit of any convolutions in $k$ space can be extended to infinity. This may be called the local approximation because it reduces equation (17) to the form of a local differential operator; see reference [17] for a discussion of the error involved in this approximation.

In keeping with the perturbation theory of reference [17], the full position dependence of $f_{R}^{\alpha}$ was retained in the terms quadratic in $\boldsymbol{P}$ in equation (17). However, in the cubic and quartic terms, $f_{R}^{\alpha}$ was replaced by the atomic distribution function $f^{\alpha}$ of the reference crystal (i.e., the heterostructure perturbation $\theta_{\boldsymbol{R}}^{\alpha}=f_{\boldsymbol{R}}^{\alpha}-f^{\alpha}$ was neglected). Thus, the coefficients of the cubic and quartic terms are given by the constants

$$
\begin{aligned}
& V_{n n^{\prime}}^{\lambda \mu \nu}=V_{n n^{\prime}}^{(\lambda \mu \nu \mid \cdot)}+V_{n n^{\prime}}^{(\lambda \mu \mid \nu)}+V_{n n^{\prime}}^{(\lambda \mid \mu \nu)}+V_{n n^{\prime}}^{(\cdot \mid \lambda \mu \nu)}, \\
& V_{n n^{\prime}}^{\lambda \mu \kappa \nu}=V_{n n^{\prime}}^{(\lambda \mu \kappa \nu \mid \cdot)}+V_{n n^{\prime}}^{(\lambda \mu \kappa \mid \nu)}+V_{n n^{\prime}}^{(\lambda \mu \mid \kappa \nu)}+V_{n n^{\prime}}^{(\lambda \mid \mu \kappa \nu)}+V_{n n^{\prime}}^{(\cdot \mid \lambda \mu \kappa \nu)},
\end{aligned}
$$

in which the various terms are just expansion coefficients that were omitted from equation (12).

Because of the general lack of symmetry of the expansion coefficients in equation (12), the noncommuting operators in equation (17) have a definite operator ordering. The term involving $V_{n n^{\prime}}^{(\lambda \mid \mu)}(\boldsymbol{x})$ has the same operator ordering as the Hamiltonian studied in the work of Mlinar et al [12], and includes terms antisymmetric in $\lambda$ and $\mu$. However, that Hamiltonian $[14,15]$ does not include any terms with the operator ordering of $V_{n n^{\prime}}^{(\lambda \mu \mid \cdot)}(\boldsymbol{x})$ and $V_{n n^{\prime}}^{(\cdot \mid \lambda \mu)}(\boldsymbol{x})$.

As might be expected from the work of Luttinger [10], equation (17) is not the same as what would be obtained by substituting $\boldsymbol{p} \rightarrow \boldsymbol{P}$ into the Hamiltonian of reference [17]. The 
position-dependent terms describing the linear response to the heterostructure perturbation do have this form, since care was taken in reference [17] to retain the asymmetric parts of these material parameters. However, the bulk coefficients derived here [such as (18), (19), and the bulk part of $V_{n n^{\prime}}^{(\lambda \mid \mu)}$ ] are asymmetric in the Cartesian indices, whereas those obtained from the expansion of $v_{G G^{\prime}}^{\alpha}(\boldsymbol{k}) \equiv v^{\alpha}\left(\boldsymbol{k}+\boldsymbol{G}, \boldsymbol{k}+\boldsymbol{G}^{\prime}\right)$ in [17] were symmetric. These asymmetric coefficients generate terms in the Hamiltonian proportional to the magnetic field, such as the dipole term in equation (20) below.

\section{Other contributions to the envelope-function Hamiltonian}

Within LDA, the self-energy of the electron is a local potential that does not couple to a static magnetic field [20]. Thus, the only other term in the Hamiltonian that depends on the magnetic field is the kinetic energy $\frac{1}{2} p^{2}$. To account for the intrinsic dipole moment of the electron, this can be written as $\frac{1}{2}(\sigma \cdot p)^{2}$, where $\sigma$ is the Pauli matrix vector; the minimal substitution $\boldsymbol{p} \rightarrow \boldsymbol{P}$ then generates the electron $g$ factor $g_{0}=2$ automatically [30].

In the Luttinger-Kohn representation, the kinetic energy produces $\boldsymbol{k} \cdot \boldsymbol{p}$ terms of the usual form [9-11, 19]; a finite-dimensional multi-band effective-mass equation can then be derived using the standard canonical transformation method of perturbation theory $[8,9,11$, 19], with the $k \cdot \boldsymbol{p}$ interaction and the heterostructure potential treated as perturbations $[17,19]$. The details are essentially the same as those given in reference [17], which will not be repeated here. The outcome is a Hamiltonian with the same qualitative structure as that shown in equation (17), but with all of the coefficients renormalized [e.g., $V_{n n^{\prime}}^{(\lambda \mu \mid \cdot)}(\boldsymbol{x}) \rightarrow \bar{V}_{n n^{\prime}}^{(\lambda \mu \mid \cdot)}(\boldsymbol{x})$ ] to account for perturbative corrections.

This Hamiltonian can then be rearranged into a more convenient form [19], which yields the effective-mass Hamiltonian

$$
\begin{aligned}
\tilde{H}_{n n^{\prime}}(\boldsymbol{x}, \boldsymbol{p})= & E_{n} \delta_{n n^{\prime}}+\left\{P_{\lambda}, \pi_{n n^{\prime}}^{\lambda}(\boldsymbol{x})\right\}+\left\{\left\{P_{\lambda} P_{\mu}\right\}, D_{n n^{\prime}}^{\{\lambda \mu\}}(\boldsymbol{x})\right\}-\mu_{n n^{\prime}}^{\lambda}(\boldsymbol{x}) B_{\lambda}(\boldsymbol{x}) \\
& +P_{\lambda} P_{\mu} P_{\kappa} C_{n n^{\prime}}^{\lambda \mu \kappa}+P_{\lambda} P_{\mu} P_{\kappa} P_{\nu} Q_{n n^{\prime}}^{\lambda \mu \kappa \nu}+W_{n n^{\prime}}(\boldsymbol{x})+\varphi(\boldsymbol{x}) \delta_{n n^{\prime}} \\
& +\partial_{\lambda} Z_{n n^{\prime}}^{\lambda}(\boldsymbol{x})+\partial_{\lambda} \partial_{\mu} Y_{n n^{\prime}}^{\lambda \mu}(\boldsymbol{x})+\left\{P_{\lambda}, \partial_{\mu} \Gamma_{n n^{\prime}}^{\lambda \mu}(\boldsymbol{x})\right\}+\left[\partial_{\mu} \Phi_{n n^{\prime}}^{\lambda \mu}(\boldsymbol{x})\right] P_{\lambda} \\
& +\hat{Z}_{n n^{\prime}}^{\lambda} \partial_{\lambda} \varphi(\boldsymbol{x})+\hat{Y}_{n n^{\prime}}^{\lambda \mu} \partial_{\lambda} \partial_{\mu} \varphi(\boldsymbol{x})+\hat{\Gamma}_{n n^{\prime}}^{\lambda \mu}\left\{P_{\lambda}, \partial_{\mu} \varphi(\boldsymbol{x})\right\}+\hat{\Phi}_{n n^{\prime}}^{\lambda \mu}\left[\partial_{\mu} \varphi(\boldsymbol{x})\right] P_{\lambda},
\end{aligned}
$$

in which $\{A, B\}=\{A B\}=\frac{1}{2}(A B+B A)$ is the symmetrized product, and $\partial_{\lambda}=\partial / \partial x_{\lambda}$ acts only on the function immediately to its right. The various coefficients in this equation are the same as those defined in [17], with some modifications to be discussed below. Therefore, the definitions given in [17] are not repeated here.

The first term $E_{n}$ is just the zone-centre energy of the reference crystal, while $\pi_{n n^{\prime}}^{\lambda}(\boldsymbol{x})$ is the position-dependent momentum matrix of the heterostructure. The Luttinger $D$ matrix [10], which is half the inverse effective mass tensor, is defined by

$D_{n n^{\prime}}^{\lambda \mu}(\boldsymbol{x})=\frac{1}{2}\left(\delta_{\lambda \mu} \delta_{n n^{\prime}}+\mathrm{i} \epsilon_{\lambda \mu \nu} \sigma_{n n^{\prime}}^{\nu}\right)+\bar{V}_{n n^{\prime}}^{(\lambda \mu \mid \cdot)}(\boldsymbol{x})+\bar{V}_{n n^{\prime}}^{(\cdot \mid \lambda \mu)}(\boldsymbol{x})+\bar{V}_{n n^{\prime}}^{(\lambda \mid \mu)}(\boldsymbol{x})$,

in which $\epsilon_{\lambda \mu \nu}$ is the antisymmetric unit tensor. As discussed above, the term $\mathrm{i} \epsilon_{\lambda \mu \nu} \sigma_{n n^{\prime}}^{\nu} / 2$ accounts for the intrinsic spin dipole moment of the electron [30]. Functions such as $\bar{V}_{n n^{\prime}}^{(\lambda \mu \mid \cdot)}(\boldsymbol{x})$ have the same form as $V_{n n^{\prime}}^{(\lambda \mu \mid)}(\boldsymbol{x})$, but include additional $\boldsymbol{k} \cdot \boldsymbol{p}$ renormalization terms that are given in [17]. However, $\bar{V}_{n n^{\prime}}^{(\lambda \mu \mid \cdot)}(\boldsymbol{x})$ is no longer symmetric: $\bar{V}_{n n^{\prime}}^{(\lambda \mu \mid \cdot)}(\boldsymbol{x}) \neq \bar{V}_{n n^{\prime}}^{(\mu \lambda \mid \cdot)}(\boldsymbol{x})$.

The symmetric part of the tensor (21) is written as $D_{n n^{\prime}}^{\{\lambda \mu\}}(\boldsymbol{x})$, while the antisymmetric part determines the effective magnetic dipole moment

$$
\mu_{n n^{\prime}}^{\lambda}(\boldsymbol{x})=\frac{\mathrm{i}}{2 c} \epsilon_{\lambda \mu \nu} D_{n n^{\prime}}^{\mu \nu}(\boldsymbol{x})
$$


This operator is interpreted as a magnetic dipole moment $[10,11,19]$ because it generates a coupling of the form $-\boldsymbol{\mu} \cdot \boldsymbol{B}$; it therefore determines the value of the effective Landé $g$ factor in the Zeeman effect for the quasiparticles (electrons or holes) described by the multi-band effective-mass Hamiltonian (20). The relationship (22) between the dipole operator and the antisymmetric part of the effective mass tensor is due simply to the fact that the components of the kinetic momentum operator $\boldsymbol{P}$ do not commute, their commutator being proportional to the magnetic field (i.e., $\left.\left[P_{\lambda}, P_{\mu}\right]=-\mathrm{i} \epsilon_{\lambda \mu \nu} B_{\nu} / c\right)$ [10, 11]. The derivation of (22) is directly analogous to the standard derivation of the intrinsic dipole moment of the electron (i.e., $g_{0}=2$ ) from the Dirac equation [30].

The symmetric terms $V_{n n^{\prime}}^{(\lambda \mu \mid \cdot)}(x)$ and $V_{n n^{\prime}}^{(\cdot \mid \lambda \mu)}(x)$ do not contribute to equation (22); the sole contribution from the nonlocal potential is the asymmetric term $V_{n n^{\prime}}^{(\lambda \mid \mu)}(\boldsymbol{x})$. Note, however, that this result depends on the initial choice of (2) as the magnetic coupling Hamiltonian. As shown in the appendix, for the generalized Peierls coupling of Ismail-Beigi et al [22], the nonlocal potential contributes nothing to the dipole moment (22).

The cubic $\left(C_{n n^{\prime}}^{\lambda \mu \kappa}\right)$ and quartic $\left(Q_{n n^{\prime}}^{\lambda \mu \kappa v}\right)$ dispersion terms are just renormalized versions of (18) and (19). The term $W_{n n^{\prime}}(\boldsymbol{x})$ represents linear and quadratic contributions to the effective potential energy, one portion of which is $V_{n n^{\prime}}^{(\cdot \cdot)}(\boldsymbol{x})$ from equation (17). The screened potential $\varphi(\boldsymbol{x})$ accounts for long-range multipole potentials generated by the electron-electron Coulomb interaction [17].

The terms $Z_{n n^{\prime}}^{\lambda}(\boldsymbol{x}), Y_{n n^{\prime}}^{\lambda \mu}(\boldsymbol{x}), \Gamma_{n n^{\prime}}^{\lambda \mu}(\boldsymbol{x})$, and $\Phi_{n n^{\prime}}^{\lambda \mu}(\boldsymbol{x})$ are short-range interface terms, the contribution from which vanishes in bulk material because the spatial derivatives $\partial_{\lambda}$ are zero there. $Z$ generates a $\delta$-like interface mixing of light and heavy holes [31], while $Y$ is similar to the Darwin term in the nonrelativistic approximation to the Dirac equation. The term $\Phi$, which is antisymmetric, is a generalized Rashba coupling [8] that can be viewed as an effective spin-orbit interaction. This is precisely the antisymmetric term that was examined in the work of Mlinar et al [12]. The $\Gamma$ term has a similar structure (i.e. an interface term that is linear in $\boldsymbol{P})$ but is symmetric.

The contributions from $\hat{Z}, \hat{Y}, \hat{\Gamma}$, and $\hat{\Phi}$ are similar to those from $Z, Y, \Gamma$, and $\Phi$, but since they involve the long-range multipole potential $\varphi(x)$, they are not as well localized at the interface [17]. These terms are not included in most envelope-function theories. However, in a quantum well or other two-dimensional system, these terms have no qualitative effect, as they merely renormalize the short-range contributions [17]. This is not strictly true in quantum wires or dots, but even in these cases it may be possible to replace the long-range contributions with renormalized short-range terms, since Zunger et al [32-34] have shown that LDA wavefunctions in a variety of nanostructures can be accurately reproduced using carefully fitted empirical pseudopotentials, which by definition have no long-range multipole terms.

\section{Conclusions and discussion}

In this paper, a first-principles multi-band effective-mass Hamiltonian was derived for latticematched semiconductor nanostructures in a slowly varying magnetic field. The theory applies to systems described by norm-conserving nonlocal pseudopotentials in the local density approximation, and is based on the magnetic coupling Hamiltonian derived by Pickard and Mauri [20]. The main result, given in equation (20), is similar to what would be obtained by applying the minimal substitution $\boldsymbol{p} \rightarrow \boldsymbol{p}+\boldsymbol{A} / \boldsymbol{c}$ to the Hamiltonian derived in [17]. However, there were also several additional terms, derived from asymmetric matrix elements of the nonlocal pseudopotential, that were not included in reference [17]. As shown in the appendix, these terms are controlled by the choice of magnetic coupling for the nonlocal pseudopotential. 
The present work provides the first direct analytical demonstration of the difference between the magnetic coupling Hamiltonians of Pickard and Mauri [20] and Ismail-Beigi et al [22]. Namely, in the former case [20], the nonlocal potential contributes to the effective magnetic dipole moment, while in the latter case [22] it does not.

Turning now to the significance of the various interface terms in the Hamiltonian (20), the work of Mlinar et al [12] has established the importance of including the generalized Rashba coupling $\Phi_{n n^{\prime}}^{\lambda \mu}(\boldsymbol{x})$. In their calculations, the magnitude of this term was estimated using the expressions given in references [14] and [15], which are equivalent to the assumption that $\Phi_{n n^{\prime}}^{\lambda \mu}(\boldsymbol{x})$ is the same as the antisymmetric part $\mathrm{i} D_{n n^{\prime}}^{[\lambda \mu]}(\boldsymbol{x})$ of the effective mass tensor (21), or that $\Phi_{n n^{\prime}}^{\sigma \tau}(\boldsymbol{x})=c \epsilon_{\sigma \tau \nu} \mu_{n n^{\prime}}^{v}(\boldsymbol{x})$. (Note that for $\Gamma_{8}$ states in zinc-blende, $\mu^{v}(\boldsymbol{x})=$ $\left[\kappa(\boldsymbol{x}) J_{v}+q(\boldsymbol{x}) J_{v}^{3}\right] / c$, where $\boldsymbol{J}$ is an angular momentum $\frac{3}{2}$ matrix, and $\kappa$ and $q$ are Luttinger parameters [10]. The calculations in [12] included only the contribution from $\kappa$.) As discussed in [17], this assumption provides a reasonable order-of-magnitude estimate, but cannot be relied upon for quantitative accuracy. Thus, the large qualitative impact of $\Phi_{n n^{\prime}}^{\lambda \mu}(\boldsymbol{x})$ discovered by Mlinar et al [12] is almost certainly a real physical effect, even though the quantitative details of their calculation may not be completely accurate.

In addition to $\Phi_{n n^{\prime}}^{\lambda \mu}(\boldsymbol{x})$, the Hamiltonian (20) also includes the interface terms $Z_{n n^{\prime}}^{\lambda}(\boldsymbol{x})$, $Y_{n n^{\prime}}^{\lambda \mu}(\boldsymbol{x})$, and $\Gamma_{n n^{\prime}}^{\lambda \mu}(\boldsymbol{x})$, which were not studied by Mlinar et al [12]. A quantitative calculation of these matrix elements is now in progress (and will be reported elsewhere), but a rough idea of their significance can be obtained from dimensional and symmetry analysis. The terms $Z_{n n^{\prime}}^{\lambda}(\boldsymbol{x})$ and $Y_{n n^{\prime}}^{\lambda \mu}(\boldsymbol{x})$ affect the boundary conditions on the envelope functions, but do not contain any direct contribution from the magnetic field. Therefore, their influence on the magnetic-field-dependent properties of nanostructures is probably not as strong as that of $\Phi_{n n^{\prime}}^{\lambda \mu}(\boldsymbol{x})$. Nevertheless, their contribution may still be significant-especially the $\delta$-function term $Z_{n n^{\prime}}^{\lambda}(x)$ [31], which is of lower order [17] than the other interface terms.

On the other hand, the term involving $\Gamma_{n n^{\prime}}^{\lambda \mu}(\boldsymbol{x})$, like $\Phi_{n n^{\prime}}^{\lambda \mu}(\boldsymbol{x})$, is linear in the vector potential. The symmetry analysis of [17] shows that this term is not present in the $\Gamma_{6}$ conduction band of zinc-blende materials, and that in the $\Gamma_{8}$ valence band it is a relativistic effect that vanishes when spin-orbit coupling is neglected. Its magnitude will therefore be smaller than that of $\Phi_{n n^{\prime}}^{\lambda \mu}(\boldsymbol{x})$, but it may be important in the description of spin-splitting effects.

Therefore, even though the calculations of Mlinar et al [12] have included what is probably the dominant magnetic-field-dependent interface term, the remaining terms are not likely to be negligible. The detailed study of these terms, however, is left to future numerical work.

\section{Acknowledgment}

This work was supported by Hong Kong UGC grant number HIA03/04.SC02.

\section{Appendix}

This appendix examines the consequences of replacing the coupling Hamiltonian of Pickard and Mauri [20] with that of Ismail-Beigi et al [22], who have proposed a generalization of the well known Peierls phase [35, 36]:

$$
\tilde{V}^{\mathrm{nl}}\left(\boldsymbol{x}, \boldsymbol{x}^{\prime}\right)=V^{\mathrm{nl}}\left(\boldsymbol{x}, \boldsymbol{x}^{\prime}\right) \exp \left(\frac{\mathrm{i}}{c} \int_{\boldsymbol{x} \rightarrow x^{\prime}} \boldsymbol{A}\left(\boldsymbol{x}^{\prime \prime}\right) \cdot \mathrm{d} \boldsymbol{x}^{\prime \prime}\right) .
$$

Unlike equation (2), this does not rely on separating $V^{\mathrm{nl}}\left(\boldsymbol{x}, \boldsymbol{x}^{\prime}\right)$ into a sum of localized atomic potentials - although, for the application considered here, it is assumed that the potential has 
this form. The line integral can be evaluated by replacing $\boldsymbol{R}$ with $\boldsymbol{x}$ in equation (5) and then expanding $\boldsymbol{A}(\boldsymbol{x})$ in a Taylor series about $\boldsymbol{x}=\boldsymbol{R}$, which gives

$$
\begin{aligned}
\int_{x \rightarrow x^{\prime}} \boldsymbol{A} \cdot \mathrm{d} \boldsymbol{x}^{\prime \prime} & =\left(\boldsymbol{x}^{\prime}-\boldsymbol{x}\right) \cdot \boldsymbol{A}(\boldsymbol{R})+\frac{1}{2}\left[\left(x_{\lambda}^{\prime}-R_{\lambda}\right)\left(x_{\mu}^{\prime}-R_{\mu}\right)-\left(x_{\lambda}-R_{\lambda}\right)\left(x_{\mu}-R_{\mu}\right)\right] \frac{\partial A_{\lambda}}{\partial R_{\mu}} \\
& -\frac{1}{2}\left(x_{\lambda}-R_{\lambda}\right)\left(x_{\mu}^{\prime}-R_{\mu}\right)\left(\frac{\partial A_{\lambda}}{\partial R_{\mu}}-\frac{\partial A_{\mu}}{\partial R_{\lambda}}\right)+\cdots .
\end{aligned}
$$

The last term, which was not present in equation (6), is proportional to the magnetic field.

If the derivation in sections 3 and 4 is now carried through in the same way as before, one finds that the last term shown in equation (13) is modified as follows:

$$
K_{\lambda} K_{\mu}^{\prime} v_{G G^{\prime}}^{(\lambda \mid \mu) \alpha} \rightarrow\left(K_{\lambda} K_{\mu}^{\prime}+\frac{i}{2 c} \epsilon_{\lambda \mu \nu} B_{\nu}\left(\boldsymbol{R}^{\alpha}\right)\right) v_{G G^{\prime}}^{(\lambda \mid \mu) \alpha}
$$

The effect of the additional term is to cancel the contribution from $V_{n n^{\prime}}^{(\lambda \mid \mu)}(\boldsymbol{x})$ to the effective dipole moment (22). Thus, for this coupling, the nonlocal potential makes no contribution to the dipole moment. A similar conclusion was reached by Kane [37], who used the Peierls coupling (A.1) for the special case of a constant magnetic field.

Therefore, different choices of coupling between the nonlocal potential and the magnetic field have a direct impact on the effective dipole moment of the electron. This provides an explicit analytical demonstration of the differences demonstrated numerically in [20].

\section{References}

[1] Kato Y K, Meyers R C, Gossard A C and Awschalom D D 2004 Science 3061910

[2] Wunderlich J, Kaestner B, Sinova J and Jungwirth T 2005 Phys. Rev. Lett. 94047204

[3] Ganichev S D and Prettl W 2003 J. Phys.: Condens. Matter 15 R935

[4] Ganichev S D, Bel'kov V V, Golub L E, Ivchenko E L, Schneider P, Giglberger S, Eroms J, De Boeck J, Borghs G, Wegscheider W, Weiss D and Prettl W 2004 Phys. Rev. Lett. 92256601

[5] Bastard G 1988 Wave Mechanics Applied to Semiconductor Heterostructures (New York: Wiley)

[6] Ivchenko E L and Pikus G E 1997 Superlattices and Other Heterostructures: Symmetry and Optical Phenomena 2nd edn (Berlin: Springer)

[7] Ivchenko E L 2005 Optical Spectroscopy of Semiconductor Nanostructures (Harrow: Alpha Science)

[8] Winkler R 2003 Spin-Orbit Coupling Effects in Two-Dimensional Electron and Hole Systems (Springer Tracts in Modern Physics vol 191) (Berlin: Springer)

[9] Luttinger J M and Kohn W 1955 Phys. Rev. 97869

[10] Luttinger J M 1956 Phys. Rev. 1021030

[11] Bir G L and Pikus G E 1974 Symmetry and Strain-Induced Effects in Semiconductors (New York: Wiley)

[12] Mlinar V, Tadić M, Partoens B and Peeters F M 2005 Phys. Rev. B 71205305

[13] Eppenga R, Schuurmans M F H and Colak S 1987 Phys. Rev. B 361554

[14] Foreman B A 1993 Phys. Rev. B 48 R4964

[15] Foreman B A 1997 Phys. Rev. B 56 R12748

[16] Bernevig B A and Zhang S C 2005 Phys. Rev. Lett. 95016801

[17] Foreman B A 2005 Phys. Rev. B 72165345

[18] Rodina A V, Efros Al L and Alekseev A Yu 2003 Phys. Rev. B 67155312

[19] Leibler L 1977 Phys. Rev. B 16863

[20] Pickard C J and Mauri F 2003 Phys. Rev. Lett. 91196401

[21] Payne M C, Teter M P, Allan D C, Arias T A and Joannopoulos J D 1992 Rev. Mod. Phys. 641045

[22] Ismail-Beigi S, Chang E K and Louie S G 2001 Phys. Rev. Lett. 87087402

[23] Foreman B A 2005 Phys. Rev. B 72165344

[24] Colombo L, Resta R and Baroni S 1991 Phys. Rev. B 445572

[25] Peressi M and Baroni S 1994 Phys. Rev. B 497490

[26] Mauri F and Louie S G 1996 Phys. Rev. Lett. 764246

[27] Kohn W 1959 Phys. Rev. 1151460 
[28] Foldy L L and Wouthuysen S A 1950 Phys. Rev. 7829

[29] Burt M G 1992 J. Phys.: Condens. Matter 46651

[30] Sakurai J J 1967 Advanced Quantum Mechanics (Reading, MA: Addison-Wesley) pp 78 and 85-6

[31] Ivchenko E L, Kaminski A Yu and Rössler U 1996 Phys. Rev. B 545852

[32] Mäder K A and Zunger A 1994 Phys. Rev. B 5017393

[33] Wang L W and Zunger A 1995 Phys. Rev. B 5117398

[34] Fu H and Zunger A 1997 Phys. Rev. B 551642

[35] Peierls R 1933 Z. Phys. 80763 (English Transl. [36])

[36] Dalitz R H and Peierls R 1997 Selected Scientific Papers of Sir Rudolf Peierls (World Scientific Series in 20th Century Physics vol 19) (Singapore: World Scientific) pp 97-119

[37] Kane E O 1958 J. Phys. Chem. Solids 6236 